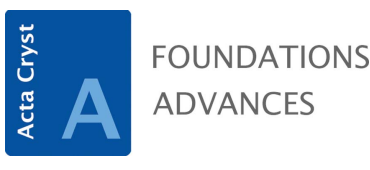

ISSN 2053-2733

\section{Geometry of Crystals, Polycrystals, and Phase Transformations. By Harshad K. D. H. Bhadeshia. CRC Press, 2018. Hardcover, Pp. xv + 251. Price GBP 37.59. ISBN 9781138070783.}

\author{
Massimo Nespolo* \\ Université de Lorraine, CNRS, CRM2, F-54000 Nancy, France. *Correspondence e-mail: \\ massimo.nespolo@univ-lorraine.fr
}

Keywords: book review; geometry of crystals; phase transformations.

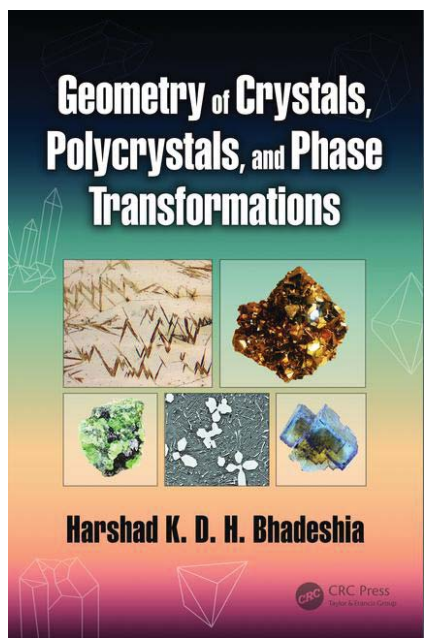

(C) 2018 International Union of Crystallography
Geometry of Crystals, Polycrystals, and Phase Transformations is a relatively small book (available also as an eBook) whose pretentious title does not faithfully represent the actual content. Indeed, the book does not address crystals in general, but metallic crystals (metals and alloys), i.e. only the higher-symmetry cases, whose geometry is particularly simple to deal with. The reader interested in a more general approach, valid for lowsymmetry crystals as well, will not find this book of much help. It clearly suffers from the bias coming from the author's background; in fact, it is hard to understand the rationale behind giving detailed (and often incorrect) definitions of basic concepts like 'equivalent', 'symmetry', 'lattice', while terms like 'Peierls barrier', 'Burgers vector', 'deviatoric' and 'shear modulus' are used without first being defined. The large number of solved exercises would certainly be a strong point of the book; unfortunately, the background necessary for solving them is not given in the text but as part of the solution, which makes the whole set of exercises unsuitable for self-study.

The text is divided into two parts, of comparable length: 'Basic crystallography' (eight chapters) and 'A few advanced methods' (five chapters), followed by two short appendices on matrix algebra. The choice of the title of the second part suggests a lack of inspiration. In fact, the titles of the chapters composing it ('Orientation relations', 'Homogeneous deformation', 'Invariant-plane strains', 'Martensite', 'Interfaces') do no fit the idea of 'methods', even 'advanced' ones. But this is far from being the only flaw of the book.

Fundamental and inexcusable crystallographic misunderstandings tessellate the path of the reader through the text. A constant, most disturbing confusion between lattice and structure pervades the whole text; for the expert reader, this is most annoying; for the beginner, it is a trap which will lead to lifelong confusions and mistakes. It is therefore not surprising to find another serious mistake in the list of acronyms with which the book begins, namely assigning the term 'trigonal' to the letter ' $\mathrm{R}$ ' (which, by the way, should have been in italics). The fundamental difference between trigonal and rhombohedral seems to be beyond the scope of this book.

Chapter 1 has the title 'Introduction and point groups' and is a short introduction to lattices, structures, symmetry operations and point groups. This chapter is a collection of mistakes and imprecise terms which should never have been written: reference to well established texts would have been a much wiser choice. Besides the confusion between 'trigonal' and 'rhombohedral' already mentioned above, we find the term 'Miller indices' also used to indicate the direction indices; crystal systems are imprecisely defined by the restrictions on cell parameters (Table 1.1), where unnecessary restrictions, unrelated to the symmetry, appear as well, although, to be fair, in a different colour (black) with respect to those imposed by symmetry (in red). Figure 1.6 shows the projection of 14 types of unit cell, which however correspond to only 12 types of Bravais lattices. In fact, the projection of a $b$-unique $m B$ unit cell (equivalent to $m P$ ) is incorrectly labelled $m C$ (the in-plane angle should have been right to get an $m C$ unit cell) and the 'trigonal' (which does not exist: read 'rhombohedral' instead) unit cell is shown without any centring nodes, which means that the $h P$ unit cell is shown twice. Miller indices (the correct ones: indices of lattice planes) are given as $h_{1}, h_{2}, h_{3}$ instead of $h, k, l$ and are incorrectly defined as never containing common factors, which is true only for primitive 
unit cells (Nespolo, 2015). The screw or glide component of the corresponding screw rotations and glide reflections are said to be 'rational fractions' of a period, which is true for the defining operation of a screw axis or glide plane, but certainly not in general (hint: how would you describe a rotation followed by an integer translation parallel to the axis and relating atoms in different unit cells?). The inversion point on a rotoinversion axis is called 'center of symmetry', although there is no such element when the rotation component is even. The too short and insufficient section on point groups gives just a few examples and contains several mistakes. The term 'crystal class' is wrongly used as a synonym of 'crystal system' (compare Tables 1.1. and 1.2). Symmetry elements ('mirror planes', 'center of symmetry', 'inversion axes' - the 'roto' must have lost its path) are mixed up with symmetry operations ('rotations'). The Hermann-Mauguin symbol of $4 \mathrm{~mm}$ pointgroup type is incorrectly interpreted in Fig. 1.9, where the two sets of crystallographically inequivalent mirror planes are attributed to the crystallographically equivalent directions ' $x$ ' and ' $y$ ' (read $a$ and $b)$. Centrosymmetric cubic groups miss the overline of the threefold rotoinversion ( 3 instead of $\overline{3}$ ); this is a common problem throughout the book.

Chapter 2 is devoted to 'Stereographic projections' and is a quite clear, although synthetic, exposition of the subject. Unfortunately, this chapter overlooks certain details too. In Fig. 2.5 neither the square brackets for directions nor the parentheses for faces are used, so one does not know what the given indices stand for. Fig. 2.7 is said to be the stereographic projection 'for the cubic system' while the symmetry elements of point-group type 432 are shown in part $(b)$; in part (a), where the parentheses are missing again, the pole of (101) is absent, and only poles of faces oriented north or vertical are given ... with the exception of (011) which would therefore completely destroy the symmetry of the projection. Part $(c)$ is the variation of the elastic modulus of body-centred cubic iron, which is cubic holohedral; why it is given in the same figure that contains the stereographic projection of 432 is left to the reader's imagination. Fig. 2.10 shows the combination of 2 and $\overline{1}$ giving $\overline{2}$, i.e. $m$, which is wrongly labelled as $2 / m$.

Chapter 3 naturally follows the previous chapter. It has the title 'Stereograms for low symmetry systems', which is totally deceiving, given that - apart from one figure where the stereographic projection of a cubic crystal is compared with that of three orthorhombic crystals with different cell parameters - only hexagonal crystals are dealt with here. It is not clear why there is a need to split the topic into two different short chapters. Bravais-Miller indices are renamed 'MillerBravais' and Weber indices (the name Weber is never used) are (unfortunately) used for directions. Note that angles between planes are not 'also the angles between their normals', as incorrectly stated, but the corresponding supplementary angles.

Chapter 4 is perhaps the shortest chapter ever written (11 pages including a few references) under the ambitious title 'Space groups'. It starts with a huge slip, where a point group is said to be a collection of symmetry elements (instead of operations). It is followed by another incorrect statement, according to which space groups include translations 'that are fractions of a repeat unit': not only do space groups exist without such translations $(P 1, P \overline{1}, P 2, P m \ldots$, the reader will easily complete the list), but when these translations exist - for example, if a centred unit cell is used, or as part of a screw rotation or glide reflection - integer translations exist as well. The finite number of space-group types and lattice types is attributed to space groups and lattices, respectively, which are both infinite in number. For a 16-line introduction to the topic, one could hardly do worse. Table 4.1 lists a subset of glide planes ( $e$ and $g$ are missing) and incorrectly calls the $n$-glide 'diamond glide'. After two pages full of imprecise statements we already leave the subject to move on to read about some structures, where further imprecisions lie in wait. For example, the 'motif' of cuprite $\left(\mathrm{Cu}_{2} \mathrm{O}\right)$, i.e. the six atoms $(4 \mathrm{Cu}$ and $2 \mathrm{O})$ in the unit cell, is said to be 'located at each lattice point'; the brief, graphic analysis of the projection of the structure concludes with the presence of a 'center of symmetry' at $\frac{1}{2}, \frac{1}{2}, \frac{1}{2}$ whereas there are eight inversion centres (if any) in every primitive unit cell. A trial-and-error procedure is used to guess possible atomic positions compatible with the formula unit, ending with a short extract of the Wyckoff position of the $P n \overline{3} m$ space-group type, where the multiplicity is called 'number'. The same quick analysis is applied to $\mathrm{Fe}_{2} \mathrm{USi}$, cementite, diamond, sphalerite, ending with another mistake in Fig. 4.8, where the intersection symmetry of $m \overline{3} \mathrm{~m}$ and $\mathrm{mmm}$ is said to be $2 / \mathrm{m}$ whereas for the given respective orientations it is clearly $\mathrm{mmm}$.

Chapter 5 deals with 'The reciprocal lattice and diffraction'. After briefly introducing the notion of reciprocal basis whose vectors are obtained as a vector product of those of the direct basis, Bragg's law is presented without giving any hint why the phenomenon of diffraction can be described in terms of reflection. Sure, it is well known, as is Bragg's law too; one should either give the reader an understandable presentation, or refer him/her to the literature. Next, we move to 'Intensities', where the structure factor appears as a deus ex machina, immediately used to obtain some systematic absences (a geometric explanation would have been more convincing, especially considering that no demonstration is meant to be given here). The Ewald sphere is introduced in Fig. 5.4, but with no mention in the text; without any discussion, it will be difficult for a beginner to understand its application to thin foils and electron diffraction in Fig. 5.11.

The last three chapters of the first part depart from basics and concentrate on topics specific to metallurgy, but the space available is limited. Given the poor job done in the first five chapters, these could have been skipped completely, replaced by a list of essential topics with relevant references provided.

Chapter 6 is devoted to 'Deformation and texture'. The chapter begins with a discussion of a slip (without defining what it is) to maximize the Schmid factor (undefined: the reader is referred to the literature), the largest shear stress on the basis of Diehl's rule. What is that? As there is no space to define or explain, we have to rush to texture, which at least gets a definition. The chapter quickly ends with less than three 
pages about orientation distribution functions and the rotations (Euler angles) needed to bring basis vectors to coincide.

We now move to Chapter 7, about 'Interfaces, orientation relationships', where grain misorientation and coincidencesite lattices (CSLs) are introduced. A quite clear and straightforward way of finding a CSL is introduced via rotation matrices, provided however that an orthonormalization step is adopted. Indeed, the general rotation matrix in equation 7.9 (whose derivation is given in Appendix B) is obtained by a similarity transformation from a cubic basis. In Fig. 7.4 all the lattice nodes centring the faces are missing so that the $c F$ unit cell looks like $c P$ and the change of basis to $t I$ seems to be using vectors pointing nowhere. The last chapter of this part deals with 'Crystallography of martensitic transformations'. With only eight pages, including the references, there is really not space to analyse the subject in depth and, anyway, it is covered in the second part of the book (why then introduce it so briefly here?). The reader is presented with the ideal Bain strain and the approximate orientation relationships between austenite and martensite, obtained by combining the Bain strain with a rigid-body rotation.

Chapter 9, 'Orientation relations', is essentially a set of solved exercises to match the axial settings of cementite and ferrite, austenite and ferrite, or of two identical crystals with an originally different orientation. It is only in Section 9.5 that we finally meet the metric tensor (renamed simply the metric'): one wonders why it is not used from the beginning? The chapter ends with a definition of cross product (why at this point?), for which the symbol of a wedge product (i.e. a bivector in exterior algebra) is used instead.

The following chapter deals with 'Homogeneous deformations', i.e. affine transformations applied to the whole crystal structure. The worked-out example of Bain strain is followed by a short introduction to eigenvalues and eigenvectors to find the principal axes and a factorization of the deformation in stretch and rotation, followed by invariant-plane strains, to define interfaces, with a brief mention of more complex deformations.

The last three chapters are those for which the respective subjects are presented with a reasonable amount of detail and represent the only part of the book worth reading. Invariant- plane strains come back as the subject of Chapter 11, where slip, deformation (mechanical) twins and stepped interfaces are presented. Some lack of rigour in the language is regrettable ('twin' is the whole heterogeneous crystalline edifice, not just an 'individual' or 'domain', as incorrectly called here); the lack of reference to the more general reticular treatment of twinning is also a defect of this chapter, which however contains a number of examples fully worked out and worth studying. The following chapter on martensite is painful reading because of the constant confusion between lattice and structure. Otherwise, it shares with the previous chapter the merit of being sufficiently detailed and rich in examples. The last chapter deals with 'Interfaces' (again? Yes!). The treatment goes through the misorientation at a grain boundary, CSLs (these too already introduced before) and their generalization as the 0-lattice and the displacement shift complete lattice, again with several examples and detailed calculations.

In summary, at the end of the journey through almost 250 pages of text, we get the strong impression that most of it consists of a collection of notes that could be useful as a support for a lecture, but whose stand-alone value is highly questionable. This impression is reinforced by the set of teaching materials available online and meant to be a companion to this book. Only the last three chapters stand out as being of reasonable book style. However, it is hard to find a reason for recommending this book: Bollmann's classical text (Bollmann, 1970) does a much better job, although one may need to complement it with some details specific to martensite. Mittemeijer's recent textbook (Mittemeijer, 2010) contains more than enough on this subject and is a much more reasonable choice to cover the whole topics presented in this book.

\section{References}

Bollmann, W. (1970). Crystal Defects and Crystalline Interfaces, xi + 254 pp. New York, Berlin, Heidelberg: Springer.

Mittemeijer, E. J. (2010). Fundamentals of Materials Science. The Microstructure-Property Relationship Using Metals as Model Systems, xxi +594 pp. Heidelberg, Dordrecht, London, New York: Springer.

Nespolo, M. (2015). J. Appl. Cryst. 48, 1290-1298. 